\title{
Are frailty scales better than anesthesia or surgical scales to determine risk in cardiac surgery?
}

\author{
Judit Kovacs ${ }^{1}$, Liviu Moraru ${ }^{1}$, Krisztina Antal ${ }^{1}$, Adrian Cioc ${ }^{2}$, \\ Septimiu Voidazan ${ }^{1}$, and Attila Szabo ${ }^{2}$ \\ ${ }^{1}$ University of Medicine and Pharmacy of Târgu Mureș, ${ }^{2}$ Department of Anesthesia and Intensive Care, Emergency \\ Clinical County Hospital, Targu Mures, Romania
}

\begin{abstract}
Background: In the last year there has been an increasing interest for using frailty scales for risk stratification of elderly patients undergoing major surgery. We planned to compare two frailty scales with risk scales already used in cardiac surgery, to study which of these scores have better prognostic value predicting postoperative outcome in open heart surgery. Methods: We conducted a prospective clinical trial, including 57 patients over 65 years. We calculated Cardiac Anesthesia Risk Evaluation score, EuroScore II, Clinical Frailty Scale, Edmonton Frail Scale for each patient and followed the postoperative complications, length of mechanical ventilation, length of stay in the intensive care unit and hospital, and in-hospital death related to these risk and frailty scores.

Results: Postoperative complications occurred in 25 patients (43.9\%), while four patients (7\%) died with multiple organ failure. All scales had low predictability for postoperative complications, but for length of mechanical ventilation we obtained positive correlations with EuroScore II, Edmonton Frail Scale and Clinical Frailty Scale. EuroScore II can also predict the length of stay in the intensive care unit. For postoperative deaths, the highest sensitivity had EuroScore II, followed by Clinical Frailty Scale and Edmonton Frail Scale.

Conclusions: EuroScore II and the frailty scales have an increased prognostic value regarding the postoperative outcome of patients (length of mechanical ventilation and in-hospital mortality), the EuroScore II can predict the length of stay in the intensive care unit as well.
\end{abstract}

Key Words: Cardiac surgery, Elderly, Postoperative complications, Risk assessment.

Corresponding author: Judit Kovacs, M.D., Ph.D.

University of Medicine and Pharmacy of Târgu Mureș, Targu Mures 540311, Aleea Carpati 45c/48, Romania

Tel: 40-726331434, Fax: 40-365405377

Email: dr.kovacsjudit@yahoo.com

ORCID: http://orcid.org/0000-0003-4108-5592

The extended results of the study (including also a group of younger patients) was presented at the 26th Conference of the Transilvanian Museum Society, April 2016, Targu Mures, Romania.

Received: March 18, 2016.

Revised: June 25, 2016 (1st); August 7, 2016 (2nd);

September 28, 2016 (3rd); October 8, 2016 (4th).

Accepted: October 11, 2016.

Korean J Anesthesiol 2017 April 70(2): 157-162

https://doi.org/10.4097/kjae.2017.70.2.157

\section{Introduction}

The number of elderly patients undergoing cardiac surgery has been increasing in the last years. Some of these patients have a good clinical course, whereas others develop severe postoperative complications, with prolonged stay in the intensive care unit, and their postoperative quality of life often remains below expectations.

The functional reserves of various organs decrease with age and vulnerability to stress increases. This decline with age is called "frailty syndrome," and does not begin at the same age in everybody. A number of medical, psychological and social factors can affect the body's physiology, such as unhealthy diet, vitamin and trace element deficiencies, lifestyle, lack of physical

(c) This is an open-access article distributed under the terms of the Creative Commons Attribution Non-Commercial License (http://creativecommons.org/ licenses/by-nc/4.0/), which permits unrestricted non-commercial use, distribution, and reproduction in any medium, provided the original work is properly cited. 
activity, and harmful job effects [1]. This syndrome has no objective diagnostic criteria, so it is difficult to assess and quantify its severity objectively.

The severity of frailty varies and is less pronounced in those with physically and intellectually active lives [1], and denying major surgery simply based on chronological age would be unfair to those in good physical condition. On the other hand, elderly subjects with increased vulnerability will probably suffer increased morbidity and mortality after surgery, with a decrease in quality of life, so the indication for surgery should always be weighed between postoperative gain and complications, and may be wise to offer less invasive alternatives or conservative treatment to these patients.

Risk scales, used to assess patients for cardiac anesthesia and surgery, often underestimate poor outcomes of the elderly, probably because the scores mainly consider comorbidities with less consideration of disabilities and functional reserves [2,3].

Many studies have tested the efficacy of frailty scales in older patients [2-6] undergoing major surgery, but only a few have compared these scores with common risk stratification scores. Therefore, we conducted a prospective clinical study to compare two frailty scales with risk scales used in cardiac surgery and anesthesia to study which of these scores has better prognostic value regarding postoperative complications, length of stay in the intensive care unit (ICU) and hospital, length of mechanical ventilation and in-hospital postoperative mortality in patients scheduled for open heart surgery.

\section{Materials and Methods}

After approval of the Ethical Committee of the Cardiovascular Surgery Clinic, we conducted a prospective clinical trial, including patients $\geq 65$ years who agreed to cooperate and who were scheduled for open heart surgery.

The anesthetist in charge calculated the Cardiac Anesthesia Risk Evaluation (CARE) score [7] and EuroScore II [8] at the preanesthetic assessment, and another researcher who was unaware of anesthetic and surgical risk scores, calculated the frailty scores using the Edmonton Frail Scale (Table 1) and the Clinical Frailty Scale (Table 2), which contain questions on lifestyle and the physical condition of patients.

We recorded patient age, body mass index, comorbidities,

Table 1. Edmonton Frail Scale [14]

\begin{tabular}{|c|c|c|c|c|}
\hline & & 0 points & 1 point & 2 points \\
\hline Cognition & Patient is asked to draw the hands of a clock in a circle to indicate ten past eleven & No error & Minor errors & Major errors \\
\hline General health & $\begin{array}{l}\text { Number of admissions to hospital in the last year? } \\
\text { How does the patient describe their health status? }\end{array}$ & $\begin{array}{c}0 \\
\text { Good }\end{array}$ & $\begin{array}{l}1-2 \\
\text { Fair }\end{array}$ & $\begin{array}{l}\geq 2 \\
\text { Poor }\end{array}$ \\
\hline $\begin{array}{l}\text { Functional } \\
\text { independence }\end{array}$ & $\begin{array}{l}\text { In how many of the following activities does the patient require help? Cooking, } \\
\text { housekeeping, laundry, shopping, transportation, telephone, managing money, } \\
\text { taking medication }\end{array}$ & $0-1$ & $2-4$ & $5-8$ \\
\hline Social support & Will someone help the patient? & Always & Sometimes & Never \\
\hline Medication & $\begin{array}{l}\text { Does the patient have } \geq 5 \text { different drugs prescribed? } \\
\text { Forget to take medication? }\end{array}$ & $\begin{array}{l}\text { No } \\
\text { No }\end{array}$ & $\begin{array}{l}\text { Yes } \\
\text { Yes }\end{array}$ & \\
\hline Nutrition & Lost weight? & No & Yes & \\
\hline Mood & Sad or depressed? & No & Yes & \\
\hline Continence & Urinary incontinence? & No & Yes & \\
\hline \multirow{3}{*}{$\begin{array}{l}\text { Self reported } \\
\text { performance }\end{array}$} & Heavy work around the house? & No & Yes & \\
\hline & Walk up and down stairs to the second floor? & No & Yes & \\
\hline & Walk $1 \mathrm{~km}$ ? & No & Yes & \\
\hline
\end{tabular}

0-5 points: not frail, 6-7 points: vulnerable, $8-9$ points: mildly frail, 10-11 points: moderately frail, 12-18 points: severely frail.

Table 2. Clinical Frailty Scale [15]

\begin{tabular}{lc}
\multicolumn{1}{c}{ Fitness of the patient } & Score \\
\hline Very fit - robust, active, energetic, exercise daily & 1 \\
Well - no active disease, but less fit (exercise 1-2 times weekly) & 2 \\
Managing well - disease symptoms well controlled & 3 \\
Vulnerable - disease symptoms not completely controlled, patient, "slowed up", but not frankly dependent & 4 \\
Mildly frail - limited dependence on others for instrumental activities of daily living & 5 \\
Moderately frail - need help for instrumental and non-instrumental activities of daily living & 6 \\
Severely frail - completely dependent on others for activities of daily living & 7 \\
\hline
\end{tabular}


type and duration of surgery, postoperative complications, length of mechanical ventilation and hospitalization in the ICU and in the hospital, and in-hospital mortality rate (postoperative death occurring before hospital discharge).

Patients were mechanically ventilated until they were fully awake, hemodynamically stable, normothermic, and met extubation criteria (no residual neuromuscular blockade and stable gas exchange). Patients were discharged from the ICU when they had no organ dysfunction requiring technical support.

Statistical analysis was performed using MedCalc ver. 12.5.0.0 (http://www.medcalc.org/download.php), and GraphPad Prism ver. 6.0 software (GraphPad, Software Inc., La Jolla, CA, USA). Discrete variables are expressed as percentages and continuous variables are expressed as mean \pm SD. The correlation between quantitative variables was assessed using Spearman's rho. To compare the predictive value of the severity scores, receiveroperating characteristic (ROC) curves were constructed and the area under the curve (AUC) was determined. A two-tailed $\mathrm{P}$ value $<0.05$ was considered significant.

\section{Results}

We analyzed 57 patients, 19 (33.3\%) women and 38 (66.7\%) men; mean age $70.2 \pm 4.3$ years, range $65-81$ years. Comorbidities, and type and length of surgical interventions are shown in Table 3. Only one patient was free of any associated disease.

The CARE and EuroScore II scores are calculated routinely during pre-anesthetic visits, but we faced some difficulties assessing frailty scores, as 6 of the 57 patients did not understand the questions asked and required repetition or exemplification, and 21 were undecided and gave different answers to the same

Table 3. Comorbidities and Type and Length of Surgical Intervention

\begin{tabular}{lc}
\hline & Number of patients \\
\hline Comorbidities & 36 \\
Arterial hypertension & 5 \\
Respiratory diseases (COPD) & 5 \\
Renal diseases & 9 \\
Liver and gastrointestinal diseases & 10 \\
Neurological disorders, stroke in history & $19 / 5$ \\
Type II diabetes/insulin-dependent & 3 \\
Malignant tumors in history & 53 \\
Others & 23 \\
Surgical interventions & 20 \\
Valve replacement & 14 \\
On-pump coronary revascularization & $124 \pm 47 \mathrm{~min}$ \\
Coronary and valvular surgery & $249 \pm 70 \mathrm{~min}$ \\
Length of cardiopulmonary bypass & \\
Length of surgical intervention &
\end{tabular}

Values are expressed as mean $\pm \mathrm{SD}$. COPD: chronic obstructive pulmonary disease. repeated question.

Postoperative complications occurred in 25 patients (43.9\%); renal dysfunction was the most frequent $(n=13)$, followed by liver $(n=8)$ and neurological $(n=6)$ dysfunction, and low cardiac output syndrome $(n=4)$. Four patients died due to multiple organ failure (mortality: 7\%).

We analyzed the prognostic value of the four scores predicting postoperative complications, and constructed ROC curves (Fig. 1, Table 4).

The AUC was higher for the EuroScore II and the Edmonton Frailty Scale (but $<0.7$ for all risk scales, meaning low predictability), and the pairwise comparison of the ROC curves showed no significant differences.

We performed a univariate Spearman's correlation test to analyze the relationships between the different scores regarding length of mechanical ventilation and length of stay in the ICU and in the hospital, because the data did not follow a Gaussian distribution (Table 5). In the results, there were significant positive correlations between EuroScore II and length of mechanical ventilation and length of stay in the ICU. Significant correlations were detected between Edmonton Frail Scale, Clinical Frailty Scale and the length of mechanical ventilation.

Therefore, we analyzed the ROC curve and AUC for postop-

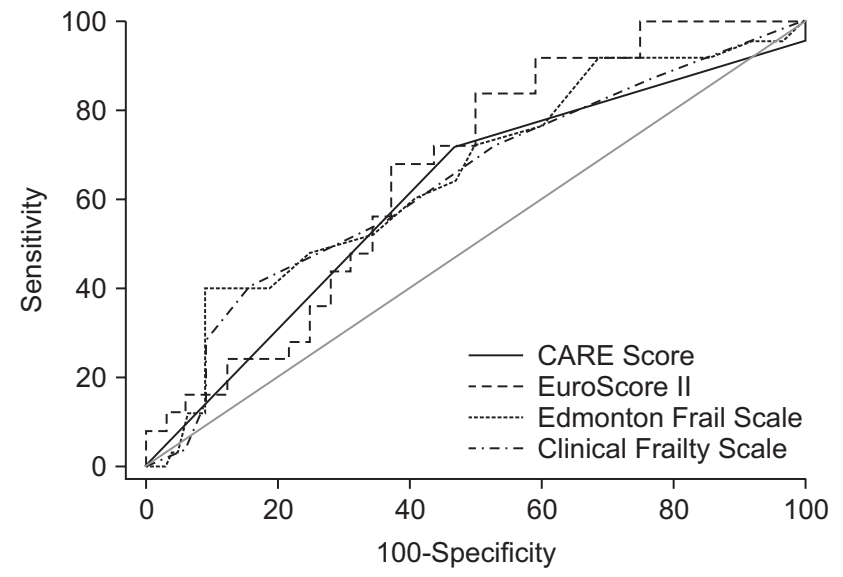

Fig. 1. Receiver operating characteristic (ROC) curves to demonstrate the ability of the risk scores and frailty scales to predict postoperative complications. CARE: cardiac anesthesia risk evaluation.

Table 4. Area Under the Curve (AUC) for Postoperative Complications

\begin{tabular}{lccc}
\hline & AUC & SE & $95 \%$ CI \\
\hline CARE Score & 0.615 & 0.0662 & $0.477-0.741$ \\
EuroScore II & 0.665 & 0.0721 & $0.528-0.785$ \\
Edmonton Frail Scale & 0.652 & 0.0745 & $0.515-0.774$ \\
Clinical Frailty Scale & 0.636 & 0.0748 & $0.498-0.760$ \\
\hline
\end{tabular}

SE: standard error, CI: confidence interval, CARE: cardiac anesthesia risk evaluation. 
Table 5. Correlations among Different Scores and the Variables Studied (Spearman's Correlation)

\begin{tabular}{|c|c|c|c|c|}
\hline \multicolumn{2}{|c|}{ Spearman's rho } & \multirow{2}{*}{$\frac{\text { Length of MV (hours) }}{0.193}$} & \multirow{2}{*}{$\frac{\text { LOS in ICU (days) }}{0.153}$} & \multirow{2}{*}{$\frac{\text { LOS in hospital (days) }}{0.177}$} \\
\hline CARE Score & Correlation coefficient & & & \\
\hline & $P$ value & 0.150 & 0.255 & 0.187 \\
\hline \multirow[t]{2}{*}{ EuroScore II } & Correlation coefficient & 0.433 & 0.329 & 0.238 \\
\hline & P value & 0.001 & 0.012 & 0.075 \\
\hline \multirow[t]{2}{*}{ Edmonton Frail Scale } & Correlation coefficient & 0.278 & 0.105 & 0.148 \\
\hline & $P$ value & 0.036 & 0.438 & 0.272 \\
\hline \multirow[t]{2}{*}{ Clinical Frailty Scale } & Correlation coefficient & 0.387 & 0.136 & 0.206 \\
\hline & $P$ value & 0.003 & 0.313 & 0.124 \\
\hline
\end{tabular}

MV: mechanical ventilation, LOS: length of stay, ICU: intensive care unit, CARE: cardiac anesthesia risk evaluation.

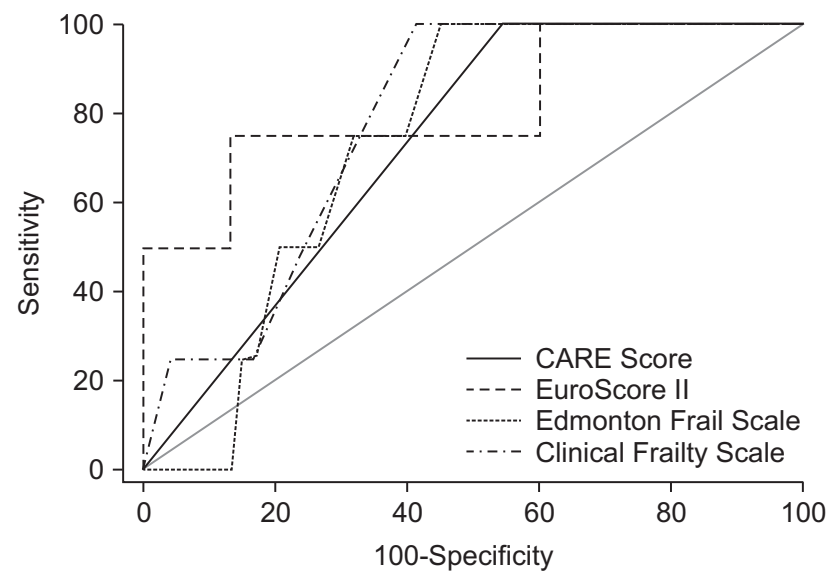

Fig. 2. Receiver operating characteristic (ROC) curves to demonstrate the ability of the risk scores and frailty scales to predict in-hospital death following cardiac surgery. CARE: cardiac anesthesia risk evaluation.

erative deaths, and found higher sensitivity for the EuroScore II (AUC 0.816), followed by the Clinical Frailty Scale (AUC 0.778) and the Edmonton Frail Scale (AUC 0.738). The pairwise comparison of the ROC curves showed no significant differences (Fig. 2, Table 6).

\section{Discussion}

More and more anesthetists and surgeons consider that the currently used risk scores underestimate postoperative risks in elderly patients with a high degree of frailty $[3,9]$. Consequently, many studies have attempted to find associations between frailty scores and postoperative morbidity and mortality in older patients undergoing major surgery. Most of these studies analyzed different frailty scales, whereas we compared two widely used scores in cardiac anesthesia (CARE and EuroScore II) and two frailty scales (Edmonton Frail Scale and Clinical Frailty Scale) that can be easily calculated.

In our study, the CARE score had the lowest sensitivity to predict postoperative complications, length of mechanical
Table 6. Area Under the Curve (AUC) for Postoperative Deaths

\begin{tabular}{llll}
\hline & AUC & SE & $95 \%$ CI \\
\hline CARE Score & 0.726 & 0.0345 & $0.592-0.836$ \\
EuroScore II & 0.816 & 0.145 & $0.691-0.906$ \\
Edmonton Frail Scale & 0.738 & 0.0804 & $0.605-0.846$ \\
Clinical Frailty Scale & 0.778 & 0.0850 & $0.649-0.878$ \\
\hline
\end{tabular}

SE: standard error, CI: confidence interval, CARE: cardiac anesthesia risk evaluation.

ventilation, lengths of stay in the ICU and the hospital, and inhospital death. The CARE score is used frequently because of its simplicity, but no exact pathologies or surgical interventions are included; some uncontrolled medical problems or types of complex surgery are exemplified, but this leaves room for clinical judgement, which means different interpretations depending on the anesthetists' or surgeons' experience.

The EuroScore II is more complex and objective than the CARE score. Nonetheless, some studies have reported that a EuroScore II $\leq 6$ overestimates mortality, and that one $\geq 13$ underestimates it $[2,6]$. In our study, the EuroScore II had the highest predictability regarding postoperative outcome (postoperative complications and deaths) of the elderly patients, length of mechanical ventilation, and length of stay in the ICU. In addition to the severity of comorbidities and the type of surgery, the EuroScore II also evaluates musculoskeletal mobility and neurocognitive dysfunction, which reflect somewhat frail patients. This is probably why the prognostic value was better for the EuroScore II than for the CARE score, and was as efficient as the frailty scales for predicting length of mechanical ventilation.

The Clinical Frailty Scale and Edmonton Frail Scale are generally considered effective and have been studied by several authors $[1,3,10]$, but some of the questions on the scales can be interpreted differently by patients from different social or cultural backgrounds, so the calculated score may over- or underestimate the actual physical and cognitive condition of the patient. In addition, the Edmonton Frail Scale is more complex and more time-consuming to perform and it contains a series 
of questions (such as social support or medication) that are less relevant to the immediate postoperative course of patients. The overall condition of a patient who is depressed or anxious will likely be overestimated by the Edmonton Frail Scale. However, both frailty scales showed good predictability for length of mechanical ventilation, which depends on the patients' muscular strength. Age-related sarcopenia and sarcopenic obesity, as well as unintentional weight loss in the elderly, lead to progressive loss of muscle mass and strength, making weaning from mechanical ventilation more difficult [11-14]. Most of the frailty scores assess the patient's fitness and muscular strength, so they are a better predictor of length of mechanical ventilation than anesthetic scores.

The Edmonton Frail Scale has been studied in elderly patients undergoing orthopedic surgery, and the score is positively correlated with the occurrence of postoperative complications and length of hospital stay [15]. We found positive correlations only with the length of mechanical ventilation and in-hospital death in patients undergoing open heart surgery.

In a study involving 400 patients $\geq 75$ years, Sündermann et al. [2] reported that patients with higher frailty scores had significantly higher mortality after major surgery. Other studies have shown that the number of complications, and lengths of stay in the ICU and in the hospital increase with increasing frailty, and that quality of life decreases in these patients during the late postoperative period [1]. Comparing the four scores, the EuroScore II (AUC 0.816) had the highest predictability for postoperative death, followed by the Clinical Frailty Scale (AUC
0.778) and the Edmonton Frail Scale (AUC 0.738), although the differences were not significant.

It is important to realize that the postoperative outcome of patients undergoing major surgery depends not only on frailty but also on the severity of any disabilities and comorbidities, which may be the reason why the EuroScore II had higher predictability than the frailty scores. Patients with high vulnerability have more comorbidities, which, in turn, can be predictors for adverse postoperative outcomes [3].

In conclusion, despite the use of different scores, it is difficult to predict postoperative complications and their severity in elderly patients, in which frailty is relatively common. The EuroScore II had higher predictability regarding the postoperative outcome of patients, length of mechanical ventilation, length of stay in the ICU and in-hospital death than the other scales, whereas the frailty scores correlated only with in-hospital death and length of mechanical ventilation, which depends on muscular strength.

A new risk stratification scoring system should be developed for elderly patients undergoing surgery, that include criteria for organ dysfunction, complexity of the surgical intervention, and an assessment of the patient's frailty and unintentional weight loss.

\section{Acknowledgments}

This study was supported by the Domus Programme of the Hungarian Academy of Sciences.

\section{References}

1. Griffiths R, Mehta M. Frailty and anesthesia: what we need to know. Contin Educ Anaesth Crit Care Pain 2014; 14: 273-7.

2. Sündermann S, Dademasch A, Praetorius J, Kempfert J, Dewey T, Falk V, et al. Comprehensive assessment of frailty for elderly high-risk patients undergoing cardiac surgery. Eur J Cardiothorac Surg 2011; 39: 33-7.

3. Rowe R, Iqbal J, Murali-Krishnan R, Sultan A, Orme R, Briffa N, et al. Role of frailty assessment in patients undergoing cardiac interventions. Open Heart 2014; 1: e000033.

4. Makary MA, Segev DL, Pronovost PJ, Syin D, Bandeen-Roche K, Patel P, et al. Frailty as a predictor of surgical outcomes in older patients. J Am Coll Surg 2010; 210: 901-8.

5. Partridge JS, Harari D, Dhesi JK. Frailty in the older surgical patient: a review. Age Ageing 2012; 41: 142-7.

6. Afilalo J, Mottillo S, Eisenberg MJ, Alexander KP, Noiseux N, Perrault LP, et al. Addition of frailty and disability to cardiac surgery risk scores identifies elderly patients at high risk of mortality or major morbidity. Circ Cardiovasc Qual Outcomes 2012; 5: 222-8.

7. Dupuis JY, Wang F, Nathan H, Lam M, Grimes S, Bourke M. The cardiac anesthesia risk evaluation score: a clinically useful predictor of mortality and morbidity after cardiac surgery. Anesthesiology 2001; 94: 194-204.

8. Noyez L, Kievit PC, van Swieten HA, de Boer MJ. Cardiac operative risk evaluation: The EuroSCORE II, does it make a real difference? Neth Heart J 2012; 20: 494-8.

9. Gogbashian A, Sedrakyan A, Treasure T. EuroSCORE: a systematic review of international performance. Eur J Cardiothorac Surg 2004; 25: 695-700.

10. Rolfson DB, Majumdar SR, Tsuyuki RT, Tahir A, Rockwood K. Validity and reliability of the Edmonton Frail Scale. Age Ageing 2006; 35: 526-9.

11. Sharma G, Goodwin J. Effect of aging on respiratory system physiology and immunology. Clin Interv Aging 2006; 1: 253-60.

12. Batsis JA, Mackenzie TA, Barre LK, Lopez-Jimenez F, Bartels SJ. Sarcopenia, sarcopenic obesity and mortality in older adults: results from 
the National Health and Nutrition Examination Survey III. Eur J Clin Nutr 2014; 68: 1001-7.

13. Kim YS, Lee Y, Chung YS, Lee DJ, Joo NS, Hong D, et al. Prevalence of sarcopenia and sarcopenic obesity in the Korean population based on the Fourth Korean National Health and Nutritional Examination Surveys. J Gerontol A Biol Sci Med Sci 2012; 67: 1107-13.

14. Dimaria-Ghalili RA, Nicolo M. Nutrition and hydration in older adults in critical care. Crit Care Nurs Clin North Am 2014; $26: 31-45$.

15. Revenig LM, Ogan K, Guzzo TJ, Canter DJ. The use of frailty as a surgical risk assessment tool in elderly patients. Curr Geriatr Rep 2014; 3 : $1-7$. 